\title{
Breast Cancer Detection with Mammogram Segmentation: A Qualitative Study
}

\author{
Samir M. Badawy ${ }^{1}$ \\ Industrial Electronics and Control Eng. Dept, \\ Faculty of Electronic Engineering, Menoufia University, \\ Egypt \\ 11rsamirb@gmail.com
}

\author{
Alaa A. Hefnawy ${ }^{2}$, Hassan E. Zidan ${ }^{3}$, and \\ Mohammed T. GadAllah ${ }^{4}$ \\ Computers and Systems Department, \\ Electronics Research Institute (ERI), \\ Egypt \\ ${ }^{2}$ alaa@eri.sci.eg, ${ }^{3}$ hbanna@eri.sci.eg, and \\ ${ }^{4}$ Mohammed.tag.1986@eri.sci.eg
}

\begin{abstract}
Mammography is specialized medical imaging for scanning the breasts. A mammography exam (A Mammogram) helps in the early detection and diagnosis of breast cancer. Mammogram image segmentation is useful in detecting the breast cancer regions, hence, better diagnosis. In this paper, we applied enhanced double thresholding-based approach for Mammograms' image segmentation. Moreover, we added the borders of the final segmented image as a contour to the original image helping physicians to easily detect the breast cancer into different Mammograms. The result is enhanced wise effect onto breast cancer qualitative detection into Mammograms, helping physicians for better diagnosis. Generalization for our study is possible for not only x-ray based Mammograms, but also for all biomedical images, as an enhanced segmentation way for better visualization, detection, and feature extraction, thus better diagnosis. Moreover, this manual thresholding method has the advantage of not only reducing processing time but also the processing storage area.
\end{abstract}

Keywords-Image processing; double thresholding segmentation; breast cancer detection into mammograms

\section{INTRODUCTION}

Breast cancer is the second common cancer worldwide after the lung cancer, the fifth common cause of cancer death, and the major cause of cancer death in women [1]. Breast cancer is the second common cancer in women after skin cancer in the U.S. Both men and women can have a breast cancer, but there are about 100 times more new cases of breast cancer in women than in men every year [2].

Beginning in the 1980s, screening mammography led to sweeping improvements in early detection of breast cancer [3]. Mammography is a specialized medical imaging that uses a low-dose $\mathrm{X}$-ray system for scanning the breasts. A mammography exam, called mammogram, helps in the early detection and diagnosis of the breast diseases in women [4].

Screening mammograms are administered to detect breast cancer in women who have no apparent symptoms. Diagnostic mammograms are used after suspicious results on a screening mammogram or after some signs of breast cancer guide the physician to check the tissue [5]. Image segmentation is an effective way for detecting the breast cancer regions in mammograms, hence, better diagnosis. Segmentation refers to the operation of partitioning an image into component parts, or into separate objects, and there is more than one approach for image segmentation [6]. More trials had been concerned with breast cancer detection by different segmentation approaches [7]-[11]. Double thresholding segmentation approach is a simple and basic way for dealing with cancer cells image segmentation [12].

In this paper the same technique of double thresholding segmentation applied in [13] and [14] for Mammograms' image segmentation was implemented. An enhancement has been done to the segmentation approach by applying some morphological operations after double thresholding. As a postprocessing the borders of the final segmented image as a contour was added to the original mammogram image helping physicians to better diagnose the breast cancer in mammograms. The proposed approach has been applied on more than one mammogram scans taken from [15].

The result is an enhanced detection and a better visualization of the breast cancer in different mammograms. Finally, it is concluded that enhanced double thresholding segmentation applied has improved wise effect onto the breast cancer detection into mammograms, helping physicians for better diagnosis. The paper is organized as follows: In Section 2 , we display the materials and methods had been used in our paper including the enhanced segmentation approach, been applied on a four different Mammograms. A brief discussion of our results is displayed in Section 3. Finally, some concluding remarks are given in Section 4.

\section{MATERIALS AND METHODS}

A four sample Mammogram images had been used in our study, every image is 1024 pixels by 1024 pixels; taken from the MiniMammographic Database [15]. The top row of Fig. 3 illustrates the four sample images included in our study in the same order presented in Table 1.

\section{A. Double Thresholding Segmentation}

Thresholding is a vital part of image segmentation, where it is required to isolate the objects from the background. Here, in this paper we used the same way of double thresholding segmentation applied in [13] and [14] for mammograms' image segmentation. 
Double Thresholding Segmentation can be illustrated simply by choosing two pixel values $\mathbf{L}$ and $\mathbf{U}$ from our image, where $\mathbf{L}$ is the lower limit of thresholding and $\mathbf{U}$ is the upper limit of thresholding, and apply the thresholding operation as: A pixel becomes white if its grey level is between $\boldsymbol{L}$ and $\boldsymbol{U}$, and black if its grey level is otherwise [6].The result is a binary (black and white) image, white for all pixels' grey levels lies in-between the two limits value $\mathbf{L}, \mathbf{U}$, and black for all the others. For every image we work on we have to determine almost the pixel values for the area we need to put the scope on, and select a better thresholding limits for better extracting for the needed regions. For the four sample mammograms included in our study, after many trials we choose $\mathrm{L}=\mathbf{0 . 7 5} \&$ $\mathrm{U}=\mathbf{0 . 9 6}$

The result of the double thresholding operation is illustrated in the second row of Fig. 3.MatLab_R2017a had been used in our study for implementing the double thresholding segmentation and the following processing including some morphological operations.

\section{B. Masking and Morphological Operations}

A specified mask had been made especially to remove the unwanted borders into the mammogram image after double thresholding (see Fig. 1).

The proposed mask is 1024 pixels by 1024 pixels. It had been designed accurately to be suitable for almost all Mammographic Image Analysis Society (MIAS)'s MiniMammographic database because the size of all its images is 1024 pixels by 1024 pixels.

Moreover, all MIAS's database images had been centered in the matrix, so the mask is suitable for both; Mammograms with right position of the breast apex and Mammograms with left position of the breast apex.

After masking, some morphological operation had been done for smoothing borders and filling holes; including: morphological opening (erosion followed by dilation), dilation, thinning, and finally filling holes. The results of the four sample images after Masking and Morphological operations are shown in the third row in Fig. 3.

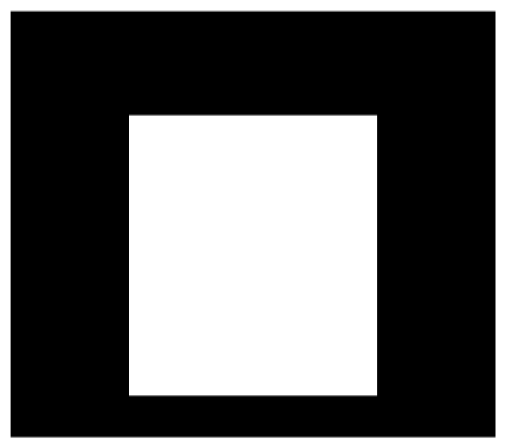

Fig. 1. The applied Mask after double thresholding.

\section{The Applied Segmentation Approach}

The Applied segmentation approach can be summarized into the flowchart shown in Fig. 2.

Step1: The original image is segmented by double thresholding producing the second row into Fig. 3.

Step2: The result of Step1; is multiplied by the mask shown in Fig. 1, after that; supposed to some morphological operations producing the third row into Fig. 3.

Step3: Contouring the abnormalities areas onto the original image by the boundaries of the resulted binary image from Step2; producing the Final Enhanced Segmented Mammogram shown in the last row of Fig. 3.

\section{RESULTS AND DISCUSSION}

Final results of the four sample mammogram images are shown in Fig. 3; where, the top row represent the original mammogram images ordered from 1 till 4 in the same order of the four Mammogram images presented into Table 1. The second row is the result of applying the double thresholding segmentation explained in Section2 in this paper. The third row is the result after masking and processing. The bottom row: final contoured images.

The final contoured images assure the wise of the applied approach into highlighting the abnormalities areas helping the physicians to easily detect the breast cancer into Mammograms. The applied enhanced double thresholding segmentation approach can be useful not only in the breast cancer detection into Mammogram scan images, but also, in extracting the regions of interest from biomedical images by selecting the appropriate threshold limit, hence helping the physicians for better diagnosis. So the generalization of our study is already possible for all biomedical images, as an enhanced segmentation way for better visualization, detection, and feature extraction, thus better diagnosis. Moreover, this manual thresholding method has the advantage of not only reducing processing time but also the processing storage area.

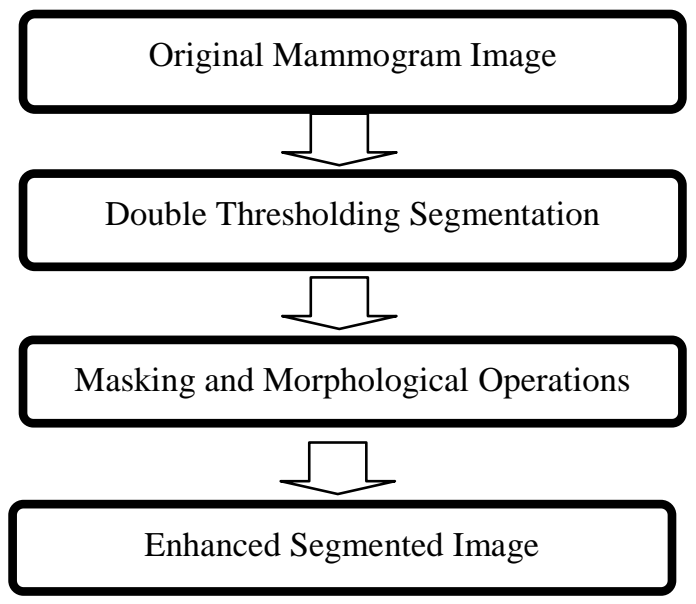

Fig. 2. Flowchart of the applied segmentation approach. 
TABLE I. DESCRIPTION OF THE FOUR SAMPLES MAMMOGRAMS BEEN USED IN OUR STUDY

\begin{tabular}{|l|l|l|l|}
\hline & Character of background tissue & Class of abnormality present \\
\hline Image 1 & Fatty-glandular & Asymmetry & Malignant \\
\hline Image 2 & Fatty & Other, ill-defined masses \\
\hline Image 3 & Dense-glandular & Architectural distortion & Malignant \\
\hline Image 4 & Fatty-glandular & Well-defined/circumscribed masses \\
\hline
\end{tabular}
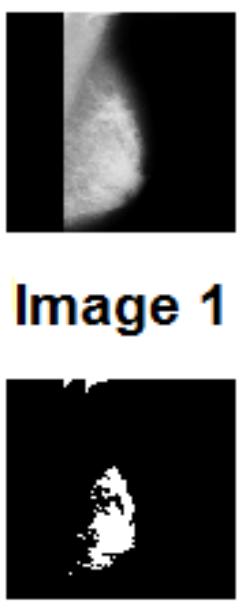

After D-Thresh

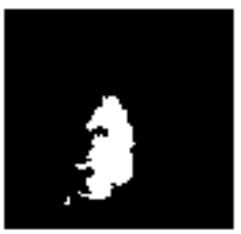

After Processing

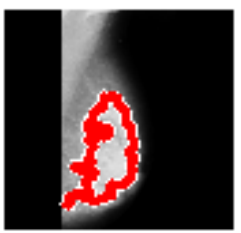

The Final Result

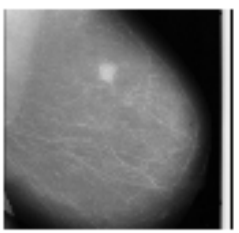

Image 2

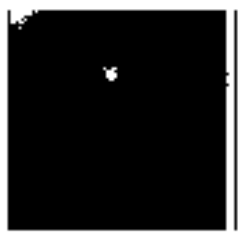

After D-Thresh

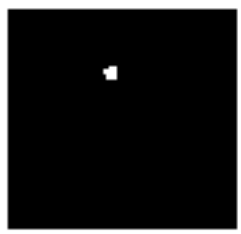

After Processing

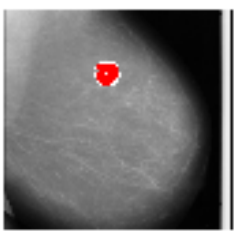

The Final Result

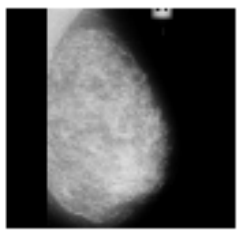

Image 3

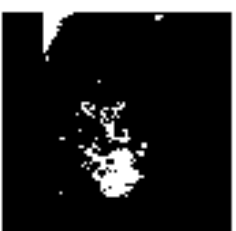

After D-Thresh

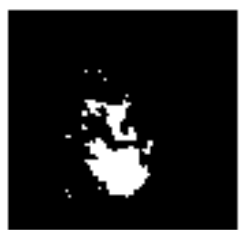

After Processing

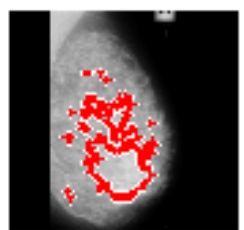

The Final Result

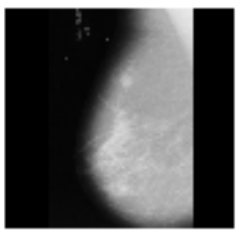

\section{Image 4}

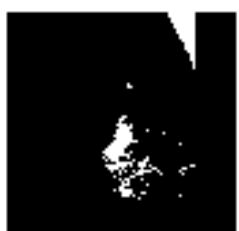

After D-Thresh

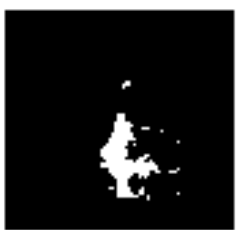

After Processing

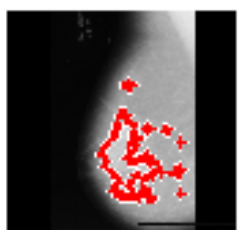

\section{The Final Result}

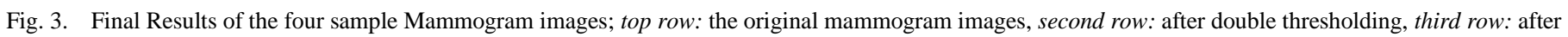
masking and processing, bottom row: final contoured images. 


\section{CONCLUSION}

In our study we proved that enhanced double thresholding segmentation applied has enhanced wise effect for breast cancer qualitative detection into Mammogram scan images, helping physicians for better diagnosis. Generalization for our study is already possible for not only $\mathrm{X}$-ray based Mammograms, but also for all biomedical images, as an enhanced segmentation way for better visualization, qualitative detection, thus better diagnosis. Moreover, this manual thresholding method has the advantage of not only reducing processing time but also the processing storage area.

\section{REFERENCES}

[1] Lisa Hutchinson, "Breast cancer: Challenges, controversies, breakthroughs", Nature Reviews Clinical Oncology, Vol. (7), pp. 669670, December 2010. Doi:10.1038/nrclinonc.2010.192. URL: https://www.nature.com/nrclinonc/journal/v7/n12/pdf/nrclinonc.2010.19 2.pdf

[2] “Overview: Breast Cancer-Patient Version", National Cancer Institute, National Institutes of Health (NIH), U.S. URL: https://www.cancer.gov/types/breast

[3] Benjamin O. Anderson, "Breast Cancer-Thinking Globally", Science, Vol. 343, Issue 6178, pp. 1403, 28 March 2014.

[4] Radiologyinfo.org, "Mammography", https://www.radiologyinfo.org/en/info.cfm?pg=mammo\#overview

[5] National Breast Cancer Foundation (NBCF), Texas, "Mammogram", URL: http://www.nationalbreastcancer.org/diagnostic-mammogram

[6] Alasdair McAndrew, “An Introduction to Digital Image Processing with Matlab Notes for SCM2511 Image Processing 1", School of Computer Science and Mathematics Victoria University of Technology, Ch. 7, pp. 137-141, Semester 1, 2004.

[7] B. K. Gayathri and P. Raajan, "A Survey of Breast Cancer Detection Based on Image Segmentation Techniques", 2016 International Conference on Computing Technologies and Intelligent Data
Engineering (ICCTIDE'16), Kovilpatti, 2016, pp. 1-5. Doi: 10.1109/ICCTIDE.2016.7725345

[8] Alaa A. Hefnawy, "An Improved Approach for Breast Cancer Detection in Mammogram based on Watershed Segmentation", International Journal of Computer Applications (0975 - 8887), Vol. 75, No.15, August 2013

[9] Abdul Qayyum and A. Basit, "Automatic breast segmentation and cancer detection via SVM in mammograms," 2016 International Conference on Emerging Technologies (ICET), Islamabad, 2016, pp. 16. Doi: 10.1109/ICET.2016.7813261

[10] M. Mustafa, N. A. Omar Rashid and R. Samad, "BreAst Cancer Segmentation Based On GVF snake," 2014 IEEE Conference on Biomedical Engineering and Sciences (IECBES), Kuala Lumpur, 2014, pp. 928-931. Doi: 10.1109/IECBES.2014.7047647

[11] R. B. Dubey, S. Bhatia, M. Hanmandlu and S. Vasikarla, "Breast Cancer Segmentation Using Bacterial Foraging Algorithm," 2013 10th International Conference on Information Technology: New Generations, Las Vegas, NV, 2013, pp. 541-545. Doi: 10.1109/ITNG.2013.88

[12] J. W. Xu, T. D. Pham and X. Zhou, "A double thresholding method for cancer stem cell detection," 2011 7th International Symposium on Image and Signal Processing and Analysis (ISPA), Dubrovnik, Croatia, 4-6 Sept. 2011, pp. 695-699.

[13] Mohamed Tarek GadAllah \& Samir Badawy, "Diagnosis of Fetal Heart Congenital Anomalies by Ultrasound Echocardiography Image Segmentation after Denoising in Curvelet Transform Domain", Online Journal on Electronics and Electrical Engineering (OJEEE), ISSN (2090-0279), Vol. (5), No. (2), pp. 554 - 560, Ref. No. : W13-E-0023, April 2013. URL: http://infomesr.org/attachments/W13-E-0023.pdf

[14] Mohammed T. GadAllah, M. M. Sharaf, Fahima A. Essawy and Samir M. Badawy, "Visual Improvement for Hepatic Abscess Sonogram by Segmentation after Curvelet Denoising", International Journal of Image, Graphics and Signal Processing (IJIGSP), vol.5, no.7, pp.9-17, DOI: 10.5815/ijigsp.2013.07.02, June 2013.

[15] J Suckling et al, "MIAS MiniMammographic Database", Mammographic Image Analysis Society (MIAS), 19 January 1995, URL: http://peipa.essex.ac.uk/info/mias.html 